Document downloaded from:

http://hdl.handle.net/10251/168878

This paper must be cited as:

Puche-Panadero, R.; Martinez-Roman, J.; Sapena-Bano, A.; Burriel-Valencia, J. (2020). Diagnosis of Rotor Asymmetries Faults in Induction Machines Using the Rectified Stator Current. IEEE Transactions on Energy Conversion. 35(1):213-221.

https://doi.org/10.1109/TEC.2019.2951008

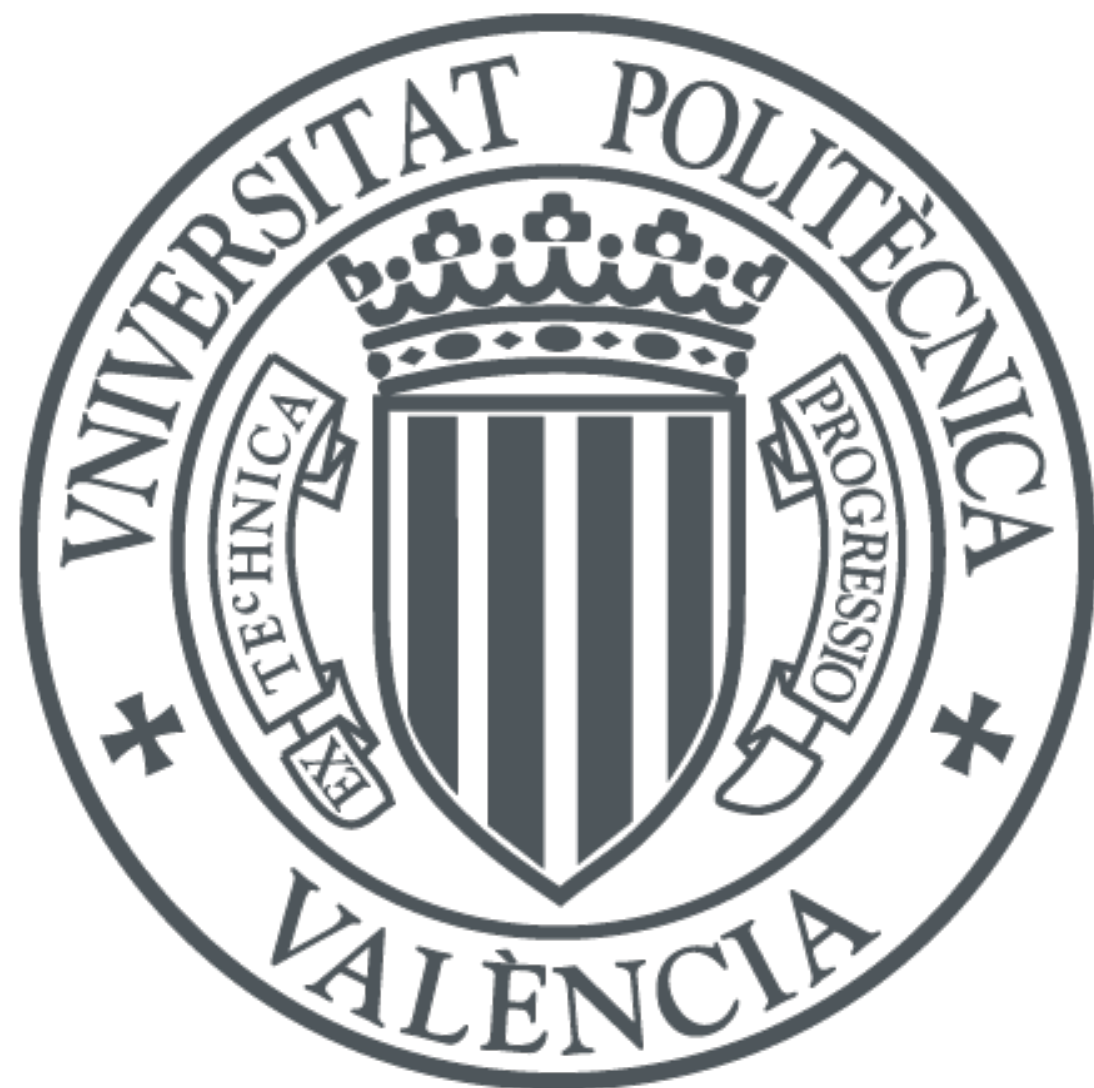

The final publication is available at

https://doi.org/10.1109/TEC.2019.2951008

Copyright Institute of Electrical and Electronics Engineers

Additional Information

(c) 2020 IEEE. Personal use of this material is permitted. Permission from IEEE must be obtained for all other uses, in any current or future media, including reprinting/republishing this material for advertising or promotional purposes, creating new collective works, for resale or redistribution to servers or lists, or reuse of any copyrighted component of this work in other works. 


\title{
Diagnosis of Rotor Asymmetries Faults in Induction Machines Using the Rectified Stator Current
}

\author{
Ruben Puche-Panadero, Member, IEEE, Javier Martinez-Roman, Angel Sapena-Bano, Jordi Burriel-Valencia.
}

\begin{abstract}
Fault diagnosis of induction motors through the analysis of the stator current is increasingly being used in maintenance systems, because it is non-invasive and has low requirements of hardware and software. Nevertheless, its industrial application faces some practical limitations. In particular, the detection of fault harmonics that are very close to the fundamental component is challenging, as in large induction motors working at very low slip, because the leakage of the fundamental can hide the fault components until the damage is severe. Several methods have been proposed to alleviate this problem, although all of them increase noticeably the complexity of the diagnostic system. In this paper, a novel method is proposed, based on the analysis of the rectified motor current. It is shown that its spectrum contains the same fault harmonics as the spectrum of the original current signal, but with a much lower frequency, and free from the fundamental component leakage. Besides, the proposed method is very easy to implement, either by software, using the absolute value of the current samples, or by hardware, using a simple rectifier. The proposed approach is presented theoretically and validated experimentally with the detection of a broken bars fault of a large induction motor.
\end{abstract}

Index Terms-Induction machines, fault diagnosis, fast Fourier transform, spectral leakage, MCSA.

\section{INTRODUCTION}

Condition based maintenance (CBM) of induction machines (IMs) is attracting extensive industrial and academic interest in recent years. IMs are a key component of modern manufacturing industry, with rated powers ranging from fractional horsepower to tenths of megawatts, and energy generation, with wind turbines up to $9.5 \mathrm{MW}$. Their ruggedness and low maintenance requirements make IMs ideally suited, in terms of reliability, for driving many industrial processes, which explains their widespread use. Nevertheless, they may fail, causing huge economical losses, well beyond the price of the machine itself. To minimize them, it is mandatory a CBM system [1]-[3] that can identify the IM faults at an early stage, allowing the optimal scheduling of repairing works, and the adjustment of the operating conditions of the IM in the meantime.

CBM of induction machines can be performed by the analysis of many different quantities [4], [5]. Among the most used, as reported in recent literature, are electrical quantities, such as machine currents [6]-[17], voltages [18] or instantaneous

This work was supported by the Spanish "Ministerio de Ciencia, Innovación y Universidades (MCIU)", the "Agencia Estatal de Investigación (AEI)" and the "Fondo Europeo de Desarrollo Regional (FEDER)" in the framework of the "Proyectos I+D+i - Retos Investigación 2018", project reference RTI2018102175-B-I00 (MCIU/AEI/FEDER, UE). The authors are with the Institute for Energy Engineering, Universitat Politècnica de València, Valencia, Spain (e-mail: rupucpa@die.upv.es,jmroman@die.upv.es, asapena@die.upv.es, jorburva@die.upv.es). power [19]; mechanical quantities, such as speed [14], torque [20], or vibrations [21]-[24]; magnetic flux [25], [26]; and thermal quantities [27], [28].

The acquired signals can be treated with a wide variety of signal processing techniques to extract fault specific features. Many of them rely on the analysis of the signal in the frequency domain, either by a direct processing using the Fourier transform (FT) [6]-[8], MUSIC [9], or spectral estimators [29]. After extracting the fault specific features, they must be further processed to establish not only the presence of a given fault but also its severity. Motor current signal analysis (MCSA) relies on predefined fault thresholds that have been analytically established and experimentally validated [8], [9], [11]-[13]. A recent trend is to use artificial intelligence (AI) methods to perform the fault detection and identification (FDDI) process, such as neural networks (ANN) [6], [20], [26], fuzzy expert system [22], or support vector machines (SVM) [18], [30]. These diagnostic methods have been applied to the detection of a wide variety of IM faults, such as rotor asymmetries [6][10], [16]-[18], [24]-[32], eccentricity [14], [19], turn-to-turn short circuits [20], [31], bearings faults [21], [22], [33], [34], or gearboxes faults [18], [30], [35].

A growing trend is the implementation of the aforementioned diagnostic techniques on low power, embedded devices, capable of monitoring online the motor condition with a reduced cost [32]. Field gate processing arrays (FPGAs) are used in [15], [23], [36], and digital signal processors (DSPs) in [37], [38]. The limited computing power and storage of lowcost devices impose severe constraints on the choice of the diagnostic methods that can be implemented for online fault diagnosis. MCSA is specially well suited to this purpose, because it has very low hardware requirements (a simple current clamp), and can be implemented using fast and efficient signal processing software, the fast Fourier transform (FFT).

In spite of these advantages, the industrial applications of MCSA faces some practical limitations. In particular, the detection of fault harmonics that are very close to the fundamental component, such as those generated by rotor asymmetries in IMs running at very low slip, is challenging [39]-[42], as in the case of large motors [43] or inverted fed induction motors [44]. In these cases, the leakage of the main component, whose amplitude is much greater than the amplitude of the fault harmonics, can hide completely them, masking the fault until it becomes severe. This is a well known problem, and several methods have been proposed to eliminate the fundamental component, using notch filters [9], extended Kalman filters [29], stochastic-resonance based adaptive filters [37], [38], matched filters [12], or time-shifting 
[34]. Nevertheless, filtering the fundamental component also attenuates the fault harmonics, distorts the spectrum, and requires a compute-intensive filtering process, that must be tuned to the exact frequency of the fundamental component, which may vary in industrial installations.

A different approach for avoiding the leakage problem is to transform the fundamental component of the current into a DC component, which produces no leakage in the discrete FT (DFT) spectrum. Several methodologies have been proposed to perform this transformation. One of them is to analyse the spectrum of the current envelope, which can be built using the Hilbert transform [16]-[18], [40], [41], or the TeagerKaiser operator [34], [45], but these transforms increase the complexity of the diagnostic algorithms, departing form the simplicity of MCSA. Other works propose the analysis of the spectrum of the square current [9], but this approach is difficult to implement using hardware multipliers [46], and also alters the relative amplitude of the fault harmonics. The extended Park's vector approach (EPVA) [47], which has proved its effectiveness for the detection of electrical and mechanical faults of IMs, can also achieve a great reduction of the fundamental component leakage, but at the cost of using a greater number of signals. A recent proposal for the demodulation of the stator current is able to demodulate also the load torque oscillations, so avoiding false positives [48].

In this paper, a novel technique, up to the authors' best knowledge, for performing MCSA with a reduced leakage of the fundamental component, is presented. It is based on the spectral analysis of the rectified current signal, a process with a very low complexity which can be performed either in hardware (a simple rectifier is needed), or in software (just keeping the absolute value of the current samples). Therefore, it can be easily implemented on low power embedded devices. Compared with previous proposals, the use of the rectified current avoids the use of lengthy transforms (Hilbert, Teager-Kaiser), maintains the relative amplitude of the fault harmonics, and avoids the leakage of the fundamental component, with the same efficiency as more complex methods. Nevertheless, it is not able to demodulate load torque oscillations, as in [48].

The structure of this work is the following one. In Section II, the Fourier transform (FT) of the rectified current signal is compared with the FT of the original current signal. In Section III the proposed method is compared with other demodulation methods presented in the technical literature, both in terms of accuracy and complexity of implementation. Section IV presents the experimental validation of the proposed method, with a large industrial motor working at a very low slip. The conclusions of this work are presented in Section V.

\section{Fault Harmonics in the Rectified Current Signal OF AN IM with Rotor ASYMMETRIES FAULtS}

A rotor asymmetry fault, such as broken bars, or asymmetries in the rotor resistances, generates an amplitude modulation in the stator current, with characteristic frequencies [40]

$$
f_{\text {asym }}=2 k s f_{1} \quad k=1,2,3 \ldots,
$$

where $f_{1}$ is the supply frequency, and $s$ is the p.u. rotor slip.
Using (1), the expression for the current of a stator phase in an IM with a rotor asymmetry is given by

$$
i(t)=I \cos \left(2 \pi f_{1} t\right)\left(1+\beta \cos \left(2 \pi f_{\text {asym }} t\right)\right),
$$

where $I$ is the maximum value of the fundamental component of the phase current, and $\beta$ is the severity of the fault, in p.u. of the fundamental component. No phase information has been included in (2) for easy of notation.

Considering only the main fault harmonic component, $k=1$ in (1), and applying trigonometrical relationships,

$$
\begin{aligned}
i(t)= & I \cos \left(2 \pi f_{1} t\right)+\frac{\beta}{2} I \cos \left(2 \pi f_{1}(1-2 s) t\right)+ \\
& +\frac{\beta}{2} I \cos \left(2 \pi f_{1}(1+2 s) t\right),
\end{aligned}
$$

where the main fault harmonics appear as side-bands of the fundamental component, at a distance $2 s f_{1}$ from it. If the slip $s$ is very small, as in the case of large motors [43], inverted fed induction motors [44], or even small motors working under low load conditions [40], this distance can be very small, which may render these fault harmonics undetected due to the leakage of the fundamental component, until the fault is severe enough. For example, in case of an incipient broken bar fault, the value of $\beta$ can be lower than $\beta=1 / 200$ [49].

The FT of (3) consists only of three spectral lines, at frequencies $f_{1},(1-2 s) f_{1}$ and $(1+2 s) f_{1}$, that is

$$
\begin{aligned}
\operatorname{FT}\{i(t)\}(f)=\hat{i}(f)= & \frac{I}{2}\left(\delta\left(f-f_{1}\right)+\right. \\
& +\frac{\beta}{2} \delta\left(f-(1-2 s) f_{1}\right)+ \\
& \left.+\frac{\beta}{2} \delta\left(f-(1+2 s) f_{1}\right)\right),
\end{aligned}
$$

where $\hat{i}(f)$ stands for the component of the FT of $i(t)$ located at frequency $f$, and $\delta$ is the Kronecker delta function. As the current signal is a real signal, only the terms of its symmetrical spectrum with positive frequency have been included in (4).

Expressing (4) in p.u. of the maximum current value, $I$, and changing to a $\mathrm{dB}$ scale gives

$$
\begin{aligned}
\hat{i}_{d B}(f)= & 0 \times \delta\left(f-f_{1}\right)+ \\
& +20 \log \left(\frac{\beta}{2}\right) \times \delta\left(f-(1-2 s) f_{1}\right)+ \\
& +20 \log \left(\frac{\beta}{2}\right) \times \delta\left(f-(1+2 s) f_{1}\right),
\end{aligned}
$$

so that the amplitude of the main fault harmonics is

$$
\left.\hat{i}\left(f_{1}+2 s f_{1}\right)\right|_{d B}=\left.\hat{i}\left(f_{1}-2 s f_{1}\right)\right|_{d B}=20 \log \left(\frac{\beta}{2}\right) .
$$

From (6) it is clear that with low values of $\beta=1 / 200$ the amplitude of the fault harmonic is only $-52.04 \mathrm{~dB}$, which difficult its reliable detection in harsh industrial environments, due to the leakage of the fundamental component.

What is proposed in this paper is the analysis of the spectrum of the rectified stator phase current, $|i(t)|$, instead of the current itself, $i(t)$. In this section it will be demonstrated theoretically that this new quantity has the same fault harmonic contents as the original $i(t)$ signal of a faulty IM, but with a much lower frequency $\left(f_{\text {asym }}\right)$ and without the leakage of the fundamental component, which improves the accuracy of the diagnostic procedure. Finally, the proposed method is very easy to implement, either taking the absolute value of the 
acquired raw $i(t)$ signal, or by rectifying it using a hardware diode bridge.

The rectified value of the current signal, $|i(t)|$, can be expressed as the original signal (2) times its sign, as

$$
|i(t)|=i(t) \times \operatorname{sgn}\left(\cos \left(2 \pi f_{1} t\right)\right)
$$

where

$$
\operatorname{sgn}(x)=\left\{\begin{array}{cl}
-1, & \text { if } x<0 \\
0, & \text { if } x=0 \\
1, & \text { if } x>0
\end{array}\right.
$$

The expression $\operatorname{sgn}\left(\cos \left(2 \pi f_{1} t\right)\right)$ in (7) is a square wave, which can be expanded as the series

$$
\operatorname{sgn}\left(\cos \left(2 \pi f_{1} t\right)\right)=\frac{4}{\pi} \sum_{h=1,3,5, . .}^{\infty}\left[\frac{\sin \left(h \frac{\pi}{2}\right)}{h} \cos \left(h 2 \pi f_{1} t\right)\right]
$$

Combining (2), (7) and (9) gives

$$
\begin{aligned}
|i(t)|= & \frac{4 I}{\pi}\left(\frac{1}{2}+\frac{\beta}{2} \cos \left(2 \pi\left(2 s f_{1}\right) t\right)+\right. \\
& +\sum_{h=3,5, . .}^{\infty}\left[\frac{\sin \left(h \frac{\pi}{2}\right)}{h} \cos \left(h 2 \pi f_{1} t\right) \times\left(\cos \left(2 \pi f_{1} t\right)+\right.\right. \\
& \left.\left.\left.+\frac{\beta}{2} \cos \left(2 \pi f_{1}(1 \pm 2 s) t\right)\right)\right]\right) .
\end{aligned}
$$

From (10) it can be observed that the rectified current signal $|i(t)|$ can be considered as the sum of three components:

- a DC component $\frac{2 I}{\pi}$.

- a low frequency component with the frequency of the main fault harmonic $\frac{2 \beta I}{\pi} \cos \left(2 \pi\left(2 s f_{1}\right) t\right)$.

- a summation of harmonic components with higher frequencies, centered around $f_{1}, 2 f_{1}, 3 f_{1}, \ldots$

If only the two first terms of $|i(t)|$ are considered, restricting the analysis of $|i(t)|$ to a low frequency band with a width greater than $2 s f_{1}$, the information about the fault harmonics is kept, avoiding the summation term in (10). From this point on, only the harmonic components in this narrow, low frequency band, will be considered from a diagnostic point of view. The resulting low frequency portion of the rectified current signal (10), designated as approximately rectified current $(\operatorname{iar}(t))$ is given by

$$
\operatorname{iar}(t)=\frac{4}{\pi} \frac{I}{2}\left(1+\beta \cos \left(2 \pi\left(2 s f_{1}\right) t\right)\right) \approx|i(t)| .
$$

The FT of (11) consists only of two spectral lines, at frequencies $0 \mathrm{~Hz}$ and $2 s f_{1}$, that is

$$
\operatorname{FT}\{\operatorname{iar}(t)\}(f)=\widehat{i} \mid(f)=\frac{4}{\pi} \frac{I}{2}\left(1 \times \delta(f)+\frac{\beta}{2} \times \delta\left(f-2 s f_{1}\right)\right)
$$

where $\widehat{|i|}(f)$ stands for the component of the FT of $i a r(t)$ located at frequency $f$. Expressing (12) in p.u. of the current maximum value, $I$, and changing to a $\mathrm{dB}$ scale, gives

$$
\widehat{|i|}|(f)|_{d B}=0 \times \delta(f)+20 \log \left(\frac{\beta}{2}\right) \times \delta\left(f-2 s f_{1}\right),
$$

with an amplitude of the main fault harmonic (in $\mathrm{dB}$ )

$$
\left.\widehat{|i|}\left(2 s f_{1}\right)\right|_{d B}=20 \log \left(\frac{\beta}{2}\right)
$$
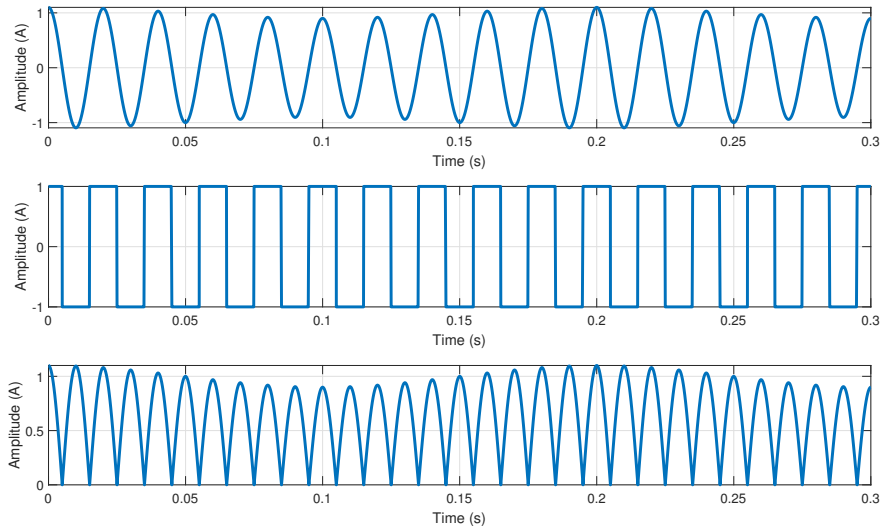

Fig. 1. Rectified current of a faulty machine, with $f_{1}=50 \mathrm{~Hz}, s=0.05$, and $\beta=0.1$. Top: synthetic current signal generated with (2). Middle: square wave of period $1 / f_{1}$ generated using the sign function. Bottom: rectified current obtained as the product of the current signal and its sign, using (7).

as shown in Fig. 1.

The comparison between the FT (in $\mathrm{dB}$ ) of the original current signal, (5), and the FT of the rectified current signal, (13), represented in Fig. 2, shows that:

- The spectral line of the fundamental component is displaced from its true frequency to a DC component.

- The two spectral lines of the main fault harmonics, which appear as side-bands of the fundamental frequency in the original current signal, are transformed into a single spectral line, at the characteristic frequency of the fault given in (1).

- The amplitudes of the fault harmonics are not altered.
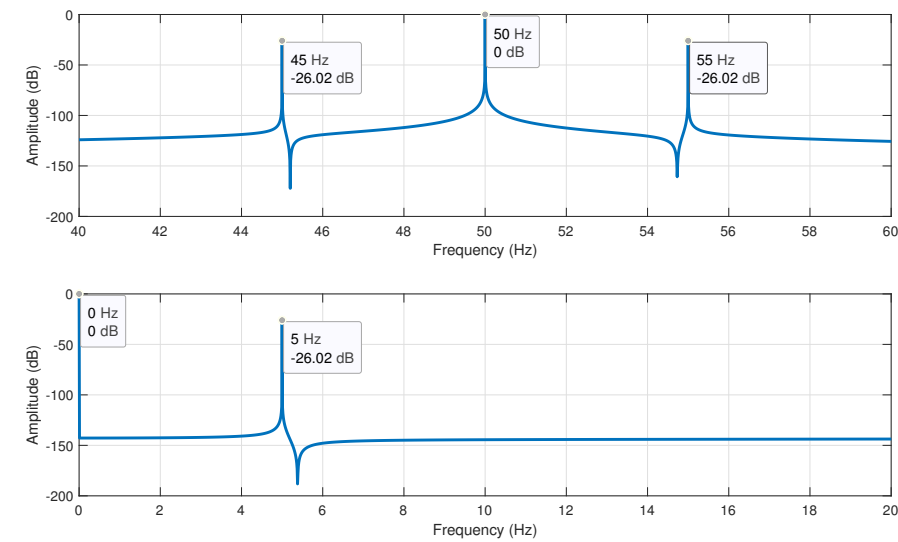

Fig. 2. Comparison between the Fourier transforms of the current signal of a machine with a rotor asymmetry (top) and the rectified current signal (bottom), using a synthetic signal with $f_{1}=50 \mathrm{~Hz}, s=0.05$, and $\beta=0.1$. The main fault harmonics appear as side-band components around the fundamental component in the case of the original current signal (top), and as a single component with the characteristic frequency of the fault in the case of the rectified current signal (bottom).

That is, the FT of the rectified current contains the same information about the fault harmonics as the original current signal, with practical advantages:

- The main fault harmonic appear as a single line, at the characteristic frequency of the fault, irrespective of the fundamental frequency, instead of two side-bands 
around the fundamental component. This fact facilitates the location of the fault harmonics in the spectrum.

- The frequency of the main fault harmonic is much lower in the spectrum of the rectified current than the frequency of the side-band fault harmonics in the spectrum of the original current signal. In this way, a much lower sampling rate can be used to acquire the rectified current signal. Nevertheless, this approach makes it necessary the use of an anti-aliasing filter.

- As the fundamental component is converted into a DC component, it has no leakage in the output of the DFT used to obtain the spectrum of the rectified current. In this way, fault harmonics with a small amplitude and very close to the main component (as in the case of IMs operating at very low slip) can be detected, while in the spectrum of the original current signal they could be buried under the leakage of the fundamental component.

To show the aforementioned advantages of the proposed method, a synthetic current signal of an IM with rotor asymmetries has been generated with (2), using the following parameters: $I=1, f_{1}=50.05 \mathrm{~Hz}, s=0.005$, and $\beta=0.01$. The sampling frequency used for generating this signal has been selected as $f_{s}=10 \mathrm{kHz}$, and the signal has been simulated during a time $t_{\text {signal }}=10$ seconds. These settings reproduce a real situation, with a fundamental frequency $(50.05 \mathrm{~Hz})$ that is not an exact multiple of the frequency resolution of the acquisition process $\left(\Delta f=1 / t_{\text {signal }}=\right.$ $0.1 \mathrm{~Hz}$ ). As can be seen in Fig. 3, top, the closest bin in the spectrum has a frequency of $50.1 \mathrm{~Hz}$, instead of $50.05 \mathrm{~Hz}$. This inability of the DFT to represent the actual value of the fundamental frequency results in a severe leakage that affects all the spectrum bins, including those corresponding to the fault harmonics. Fig. 3, top, shows the spectrum obtained with this current signal, using a Hanning window, and Fig. 3, bottom, shows the spectrum of the rectified signal, without any smoothing window. As indicated previously, the fault harmonics are nearly indiscernible in the spectrum of the current signal, because they are very close to the fundamental component, and have a small amplitude, so that they are nearly completely buried under the leakage of the fundamental. On the contrary, the fault harmonic appears clearly in the spectrum of the rectified signal, with its correct amplitude.

\section{CRITICAL COMPARISON WITH OTHER Demodulation TeChNiQues}

In this section, the proposed method is compared with other demodulation techniques reported in the technical literature, the modulus of the analytic current signal, the EPVA, the Teager-Kaiser energy operator, and the square current, in terms of accuracy and complexity of implementation.

\section{A. Modulus of the Analytic Signal of the Current}

The modulus of the analytic signal of the current has been used for the detection of single [16], [40], [41] and multiple [17] broken bars failures in motors operating at a very low
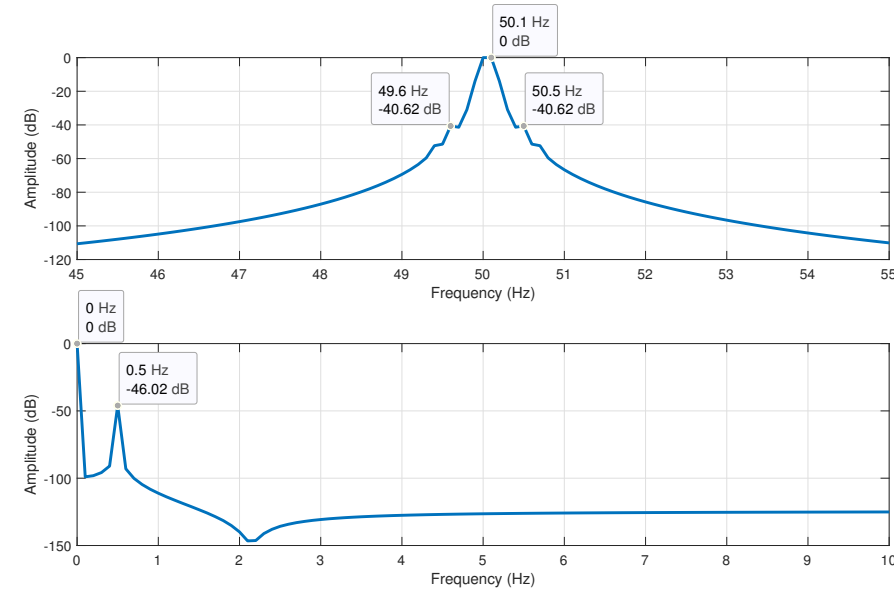

Fig. 3. Comparison between the Fourier transform of the current signal of a machine with a rotor asymmetry (top) and the rectified current signal, using a synthetic signal in (2) with $f_{1}=50.05 \mathrm{~Hz}, s=0.005$, and $\beta=0.01$, and 10 seconds of simulated current. The main fault harmonics are nearly buried under the leakage of the fundamental component in the spectrum of the current signal, even with the use of a Hanning window (top). On the contrary, the fault harmonic appears clearly in the spectrum of the rectified signal, with its correct amplitude (bottom).

slip. It is based on the analytic signal of the stator current, defined as the complex signal

$$
\operatorname{AS}\{i(t)\}=\vec{i}_{A S}=i(t)+\mathrm{j} \cdot \operatorname{HT}\{\mathrm{i}(\mathrm{t})\},
$$

where AS stands for the analytic signal, and $\operatorname{HT}\{i(t)\}$ is the Hilbert transform of $i(t)$ [17]. The $\operatorname{AS}\{i(t)\}$ can be obtained by zeroing the negative frequencies of the spectrum of $i(t)$, and doubling its DC value. The AS of the stator current of an IM with rotor asymmetries (2) can be found by applying (2) to (15) [40], giving

$$
\vec{i}_{A S}(t)=I\left(1+\beta \cos \left(2 \pi\left(2 s f_{1}\right) \cdot t\right)\right) \cdot e^{j 2 \pi f_{1} t} .
$$

The fault diagnosis procedure analyses the modulus of (16), $I\left(1+\beta \cos \left(2 \pi\left(2 s f_{1}\right) \cdot t\right)\right)$, which contains only a DC component of value $I$, and a low frequency fault harmonic at $2 s f_{1}$, with an amplitude (in $\mathrm{dB}$ ) of

$$
\left.\left|\widehat{\overrightarrow{i_{A S}}}\right|\left(2 s f_{1}\right)\right|_{d B}=20 \log \left(\frac{\beta}{2}\right) \text {. }
$$

\section{B. Extended Park's Vector Approach}

The EPVA uses the three stator currents of a three-phase IM, $i_{a}(t), i_{b}(t)$ and $i_{c}(t)$, for building the current Park's vector, defined as the complex signal [47]

$$
\vec{i}_{P V}(t)=i_{D}(t)+\mathrm{j} \cdot \mathrm{i}_{\mathrm{Q}}(\mathrm{t}),
$$

where

$$
\begin{gathered}
i_{D}(t)=\frac{\sqrt{2}}{\sqrt{3}} i_{a}(t)-\frac{1}{\sqrt{6}} i_{b}(t)-\frac{1}{\sqrt{6}} i_{c}(t), \\
i_{Q}(t)=\frac{1}{\sqrt{2}} i_{b}(t)-\frac{1}{\sqrt{2}} i_{c}(t) .
\end{gathered}
$$

The modulus of (18) is used for fault diagnosis of rotor asymmetries in IMs. In the case of a faulty machine, with 
$i_{a}(t)=i(t)(2)$, and the other two current phases forming a three phase balanced system, it is given by

$\left|\vec{i}_{P V}\right|=\left|i_{D}(t)+\mathrm{j} \cdot i_{Q}(t)\right|=\frac{\sqrt{3}}{\sqrt{2}} \cdot I\left(1+\beta \cos \left(2 \pi\left(2 s f_{1}\right) \cdot t\right)\right)$.

The modulus of the Park's vector (21) contains only a DC component of value $\frac{\sqrt{3}}{\sqrt{2}} \cdot I$, and a low frequency fault harmonic at $2 s f_{1}$, with an amplitude (in $\mathrm{dB}$ )

$$
\left.\left|\widehat{\vec{i}_{P V}}\right|\left(2 s f_{1}\right)\right|_{d B}=20 \log \left(\frac{\beta}{2}\right) \text {. }
$$

\section{Teager-Kaiser Energy Operator}

The application of the Teager-Kaiser energy operator (TKEO) to the current signal for fault diagnosis has been proposed in [45]. The TKEO of a signal $x(t)$ is given by

$$
\operatorname{TKEO}\{x(t)\}=\dot{x}(t)^{2}-x(t) \cdot \ddot{x}(t) .
$$

Applying (23) to the current signal (2), and taking only the low frequency components, gives

$$
\begin{aligned}
\operatorname{TKEO}\{i(t)\}= & i_{T K}(t)=\left(2 \pi f_{1} I\right)^{2} . \\
& \cdot\left(1+\left(1+s^{2}\right) 2 \beta \cos \left(2 \pi\left(2 s f_{1}\right) \cdot t\right)\right) .
\end{aligned}
$$

The signal $i_{T K}(t)$ (24) contains only a DC component of value $\left(2 \pi f_{1} I\right)^{2}$, and a low frequency fault harmonic at $2 s f_{1}$, with an amplitude (in $\mathrm{dB}$ ) of

$$
\left.\widehat{i_{T K}}\left(2 s f_{1}\right)\right|_{d B}=20 \log \left(\left(1+s^{2}\right) \beta\right) \approx 20 \log (\beta),
$$

for small values of $s$.

\section{Square Current Signal}

The spectrum of the square current [46] contains low frequency components which, for an IM with a rotor asymmetry fault (2), are given by

$$
\begin{aligned}
i(t)^{2}=\frac{I^{2}}{2} \cdot\left(\left(1+2 \beta^{2}\right)\right. & +2 \beta \cos \left(2 \pi\left(2 s f_{1}\right) \cdot t\right)+ \\
+ & \left.\beta I^{2} \cos \left(4 \pi\left(2 s f_{1}\right) \cdot t\right)\right) .
\end{aligned}
$$

The signal $i(t)^{2}$ (26) contains a DC component of value $\frac{I^{2}}{2}\left(\left(1+2 \beta^{2}\right)\right)$, a low frequency fault harmonic at $2 s f_{1}$, and other one at $4 s f_{1}$. In $\mathrm{dB}$, the amplitude of the spectral line corresponding to the first fault harmonic is

$$
\left.\widehat{i^{2}}\left(2 s f_{1}\right)\right|_{d B}=20 \log \left(\frac{\beta}{1+2 \beta^{2}}\right) \approx 20 \log (\beta),
$$

for small values of $\beta$.

\section{E. Comparative Analysis of the Demodulation Techniques}

The proposed method produces a main fault harmonic whose amplitude (6) is the same as in the original current signal (14), as well as the demodulation methods based on the AS (17) and the EPVA (22). On the contrary, the methods based on the TKEO (25) and the square current method (27) produce fault harmonics with an amplitude corresponding to two times the actual severity of the fault, $\beta$. Besides, the square current generates an spurious fault harmonic with a frequency equal to two times the main fault harmonic frequency, as seen in (26). All the analysed demodulation methods generate a fault harmonic with a frequency close to zero, and they are free from the leakage of the fundamental component. Fig. 4 compares the spectra of a synthetic current of a faulty machine $\left(f_{1}=50 \mathrm{~Hz}, s=0.004, \beta=0.005\right)$, obtained with the original current signal (Fig. 4.a), the proposed, rectified current signal (Fig. 4.b), the modulus of the analytic current signal (Fig. 4.c), the EPVA (Fig. 4.d), the TKEO of the current signal (Fig. 4.e), and the square current signal (Fig. 4.f). It is worth mentioning that the FFT of the TKEO of the current has the highest noise floor among the methods compared, but it is practically flat, which facilitates the detection of the fault harmonics.
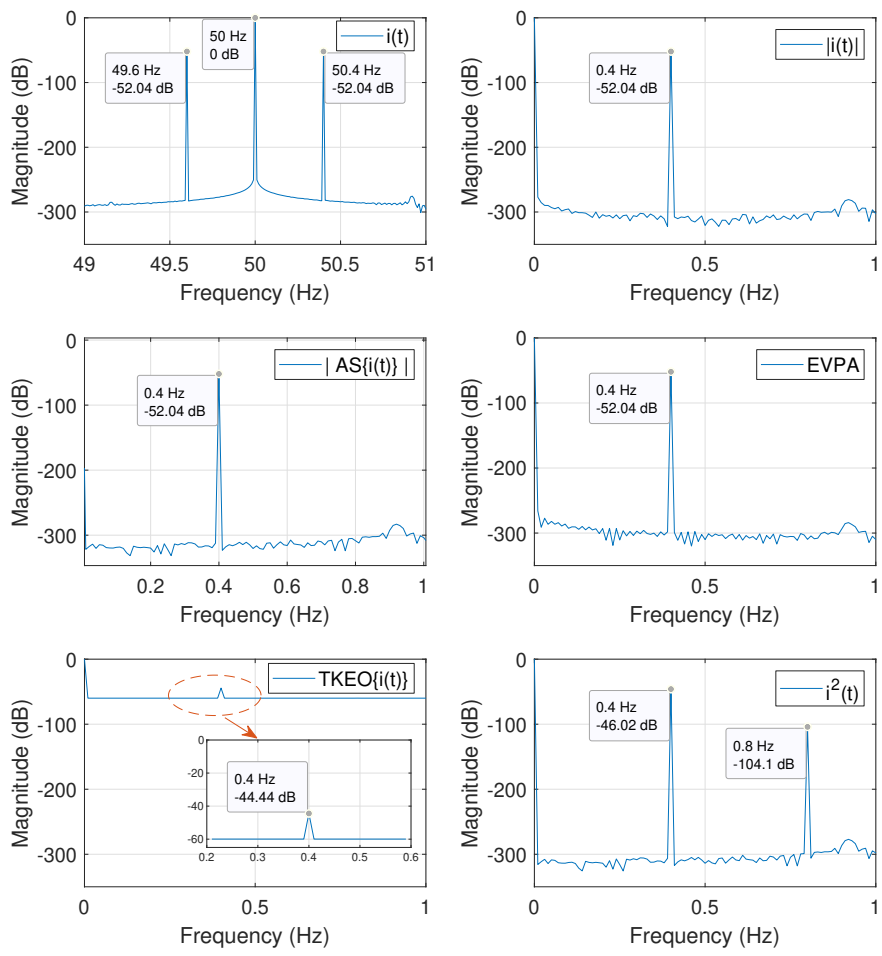

Fig. 4. Comparison of the main fault harmonics generated by different demodulation methods, using a synthetic current of a faulty machine, with $f_{1}=50 \mathrm{~Hz}, s=0.004$, and $\beta=0.005$. These spectra have been obtained with: a) the original current signal, b) the proposed, rectified current signal, c) the modulus of the analytic current signal, d) the EPVA, e) the TKEO of the current signal, and f) the square current signal.

In terms of computational effort, the proposed method is the most efficient, because it only needs a change in the sign of the negative current samples. On the contrary, the square current method needs a multiplication for every sampled value. The algorithm that implements the TKEO of the current uses two multiplications and two additions, and needs three consecutive current samples to be computed. The AS method needs to perform a Hilbert transform, followed by a modulus computation (two multiplications, one addition and one square root). The EPVA needs, additionally, to sample two or three stator currents. Therefore, among the methods compared in this work, the proposed method can be considered as the simplest one for the diagnosis of rotor asymmetries 
faults in inductions motors, and so the most suitable for being implemented on low cost embedded diagnostic devices. Additionally, the proposed method can be implemented very easily in hardware, because it only needs a full wave bridge rectifier. On the contrary, the square current method requires a multiplier integrated circuit [46], and the other methods require far more complex hardware for demodulating the current, such as Hilbert filters [32].

\section{EXPERIMENTAL VALIDATION}

The experimental validation is carried out with a large, medium voltage induction motor $(3.15 \mathrm{MW}, 6 \mathrm{kV})$, with a very low rated slip $(s=0.006)$. The proposed method is able to detect the fault harmonics given in (1) at a very low slip, as seen in the experimental tests shown in this section, while traditional MCSA fails in case of very low slip conditions. For validation purposes, the spectra obtained with the proposed method are compared with those obtained with the AS, and with the EPVA. They are practically equal to the spectrum of the rectified current, in spite of its simplicity.

It is worth mentioning that the proposed method is a general one, and can be applied to a wide variety of IMs under different supply and load conditions. Nevertheless, for the experimental validation, it has been selected a medium voltage motor, fed from the mains, because in this case the faults in the rotor cage are more prevalent than in case of motors of small size, due to the greater thermal stresses [1]. Besides, large motors in the industry are commonly fed directly from the mains, because VSDs are either very expensive for the powers involved or their complexity reduces prohibitively the reliability of the whole system [43].

The analysed motor is working in a power plant, in a harsh, noisy industrial environment, shown in Fig. 5. The characteristics of the motor are given in Appendix A. This IM had a rotor broken bar, confirmed by visual inspection of the rotor, as shown in Fig. 6. Another IM with the same characteristics is installed in the factory, without being reported any anomaly. Therefore, it has been assumed to be in healthy condition. The stator current of both motors has been acquired during 100 seconds, using a sampling frequency of $5 \mathrm{kHz}$.

The first test has been performed with the faulty motor, working at low load conditions, with a motor slip of $s=0.0018$. Traditional MCSA has been performed using a Hanning window, and the spectrum of the windows stator current is shown in Fig. 7.a. Under this conditions, the fault harmonics are barely distinguishable in the spectrum of the stator current. On the contrary, the spectrum of the rectified current (Fig. 7.b), as proposed in this work, shows clearly not only the main fault harmonic, $k=1$ in (1), but also the second fault harmonic, $k=2$ in (1), without needing any smoothing window. More complex methods as the analytic current signal (Fig. 7.c), and the EPVA (Fig. 7.d), give the same results.

The second test has been performed with the faulty motor, working at practically no load conditions, with a motor slip of only $s=0.0009$. Traditional MCSA has been performed using a Hanning window, and the spectrum of the windows stator current is shown in Fig. 8.a. Under these conditions, the fault

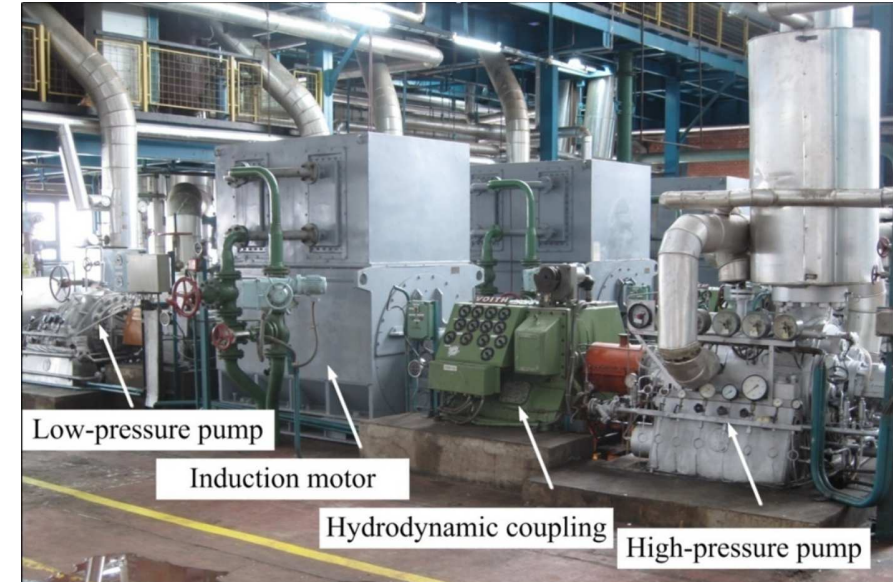

Fig. 5. The 3.15 MW motor used for validating the proposed method, installed in the thermal power plant-heating plant.
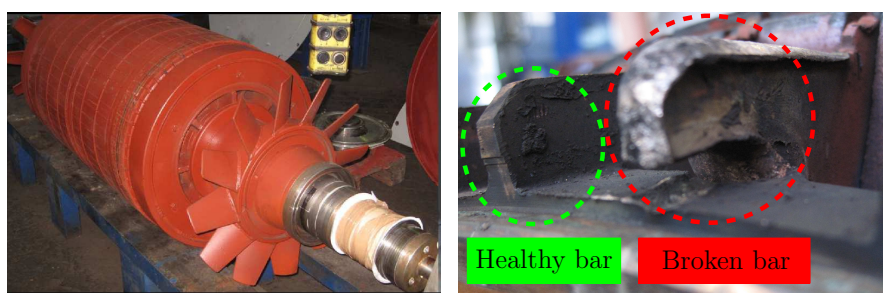

Fig. 6. Rotor of the high-rated, medium-voltage IM given in Appendix A (left), and detail of the rotor broken bar (right), used in the experimental validation of the proposed method.

harmonics do not appear in the spectrum of the stator current, due to the leakage of the fundamental component, giving a false diagnostic of healthy motor condition. On the contrary, the spectrum of the rectified current (Fig. 8.b), as proposed in this work, shows clearly not only the main fault harmonic, $k=1$ in (1), but also the second fault harmonic, $k=2$ in (1), without needing any smoothing window. The spectra obtained with the more complex methods of the analytic signal of the current (Fig. 8.c), and the EPVA (Fig. 8.d), are practically the same as the spectrum obtained with the proposed method.

The third test has been performed with the healthy motor, shown in Fig. 9. In this case there is no presence of any fault harmonic in the spectra, which indicates a healthy motor condition. Nevertheless, using traditional MCSA alone, Fig. 9.a, this assessment may not be conclusive, because the fault harmonics could be buried under the leakage of the fundamental component.

\section{Conclusions}

In this paper, a novel method has been presented for the diagnosis of rotor asymmetries in induction motors, the spectrum of the rectified stator current. It has been shown that this spectrum contains the information about the fault harmonics, which are displayed at their characteristic fault frequencies, instead of side-bands around the fundamental component. The proposed method shares the same advantages as other demodulation methods presented in the technical literature, such as the elimination of the leakage of the fundamental component, 
a)

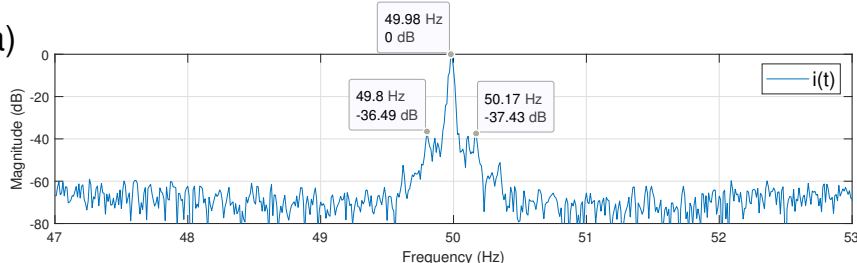

b)

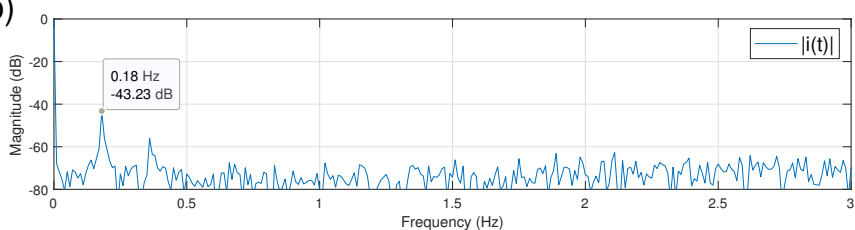

c)

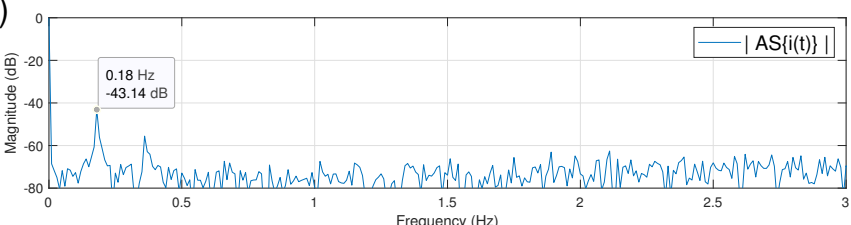

d)

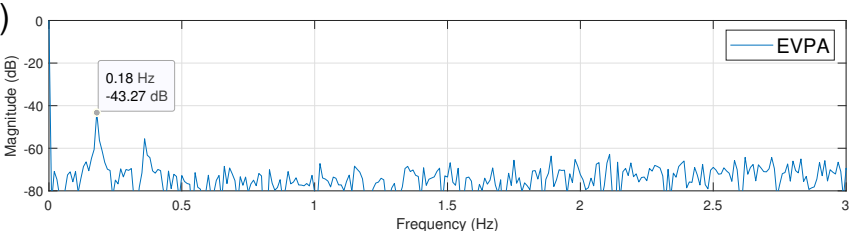

Fig. 7. Spectra of the faulty motor (one broken bar) given in Appendix A, for a motor slip of $s=0.0018$ (low load regime). These spectra have been obtained with the measured current (a), the proposed, rectified current (b), the modulus of the analytic signal of the current (c), and the EPVA (d). In the spectrum of the current (a) the fault harmonics are barely distinguishable. On the contrary, the three demodulated currents shows clearly not only the main fault harmonic, $k=1$ in (1), but also the second fault harmonic, $k=2$.

but is much simpler, in terms of computing complexity (a fast absolute value operation) or hardware implementation (a simple rectifier), as has been demonstrated theoretically and experimentally in this work. Unlike conventional MCSA, the proposed method is able to detect rotor asymmetries faults in large IMs working at a very low slip, with a low cost diagnostic system. In this paper, the proposed method has been applied to a large, industrial motor, working in stationary regime. The extension of the method to transient conditions, and its application to demodulate also the load torque oscillations, is a work in progress.

\section{APPENDIX A}

Three-phase induction motor, star connection. Rated characteristics: $P=3150 \mathrm{~kW}, f=50 \mathrm{~Hz}, U=6 \mathrm{kV}$, $I=373 \mathrm{~A}, n=2982 \mathrm{rpm}$, and $\cos \varphi=0.92$.

\section{REFERENCES}

[1] S. Kumar, D. Mukherjee, P. K. Guchhait, R. Banerjee, A. K. Srivastava, D. N. Vishwakarma, and R. K. Saket, "A comprehensive review of condition based prognostic maintenance (cbpm) for induction motor," IEEE Access, vol. 7, pp. 90690-90 704, 2019.

[2] M. Hossain, A. Abu-Siada, S. Muyeen, M. L. Hossain, A. Abu-Siada, and S. M. Muyeen, "Methods for Advanced Wind Turbine Condition Monitoring and Early Diagnosis: A Literature Review," Energies, vol. 11, no. 5, p. 1309, 52018.

[3] Y. Liu and A. M. Bazzi, "A review and comparison of fault detection and diagnosis methods for squirrel-cage induction motors: State of the art," ISA Transactions, vol. 70, pp. 400-409, 92017. a)

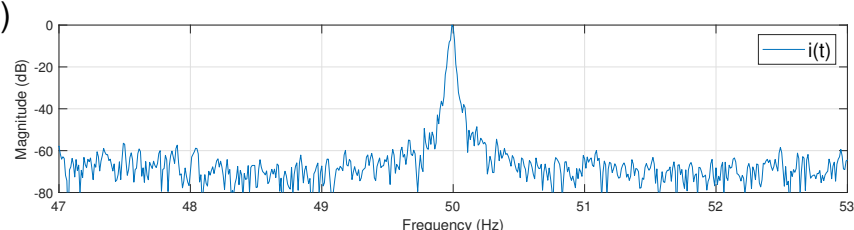

b)

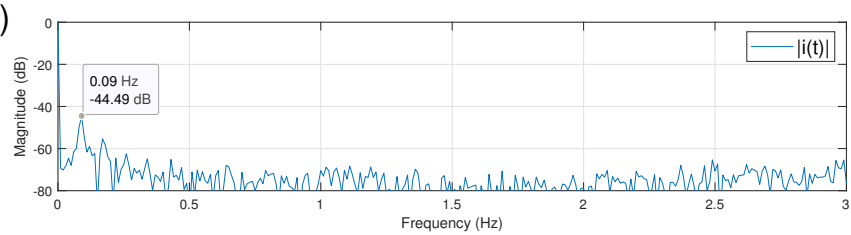

C)

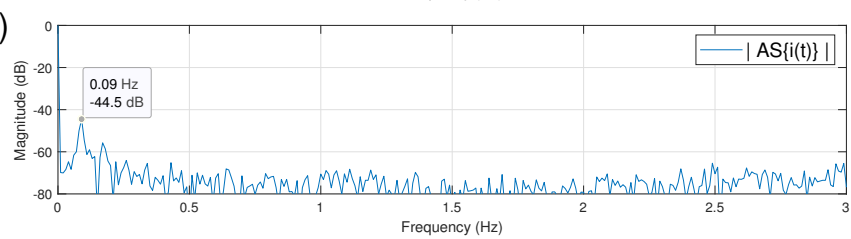

d)

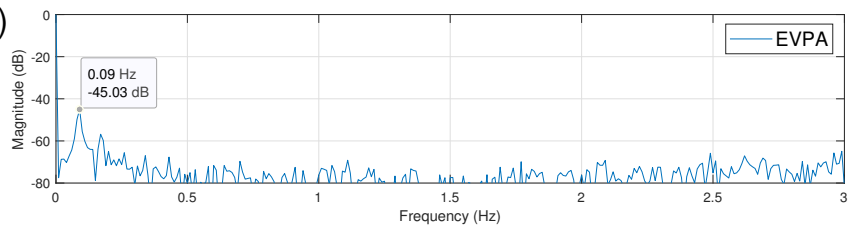

Fig. 8. Spectra of the faulty motor (one broken bar) given in Appendix A, for a motor slip of only $s=0.0009$, practically unloaded. These spectra have been obtained with the measured current (a), the proposed, rectified current (b), the modulus of the analytic signal of the current (c), and the EPVA (d). In the spectrum of the current (a) the fault harmonics are not distinguishable, due to the leakage of the fundamental component, giving a false diagnostic of healthy motor condition. On the contrary, the three demodulated currents shows clearly not only the main fault harmonic, $k=1$ in (1), but also the second fault harmonic, $k=2$.

[4] Y. Merizalde, L. Hernández-Callejo, O. Duque-Perez, Y. Merizalde, L. Hernández-Callejo, and O. Duque-Perez, "State of the Art and Trends in the Monitoring, Detection and Diagnosis of Failures in Electric Induction Motors," Energies, vol. 10, no. 7, p. 1056, 72017.

[5] H. Henao, G.-A. Capolino, M. Fernandez-Cabanas, F. Filippetti, C. Bruzzese, E. Strangas, R. Pusca, J. Estima, M. Riera-Guasp, and S. Hedayati-Kia, "Trends in Fault Diagnosis for Electrical Machines: A Review of Diagnostic Techniques," IEEE Industrial Electronics Magazine, vol. 8, no. 2, pp. 31-42, 62014.

[6] I. Martin-Diaz, D. Morinigo-Sotelo, O. Duque-Perez, and R. J. RomeroTroncoso, "An Experimental Comparative Evaluation of Machine Learning Techniques for Motor Fault Diagnosis Under Various Operating Conditions," IEEE Trans. Ind. Appl., vol. 54, no. 3, pp. 2215-2224, 52018.

[7] L. A. Trujillo-Guajardo, J. Rodriguez-Maldonado, M. A. Moonem, and M. A. Platas-Garza, "A Multiresolution Taylor-"Kalman Approach for Broken Rotor Bar Detection in Cage Induction Motors," IEEE Trans. Instrum. Meas., vol. 67, no. 6, pp. 1317-1328, 62018.

[8] D. Zappalá, N. Sarma, S. Djurović, C. Crabtree, A. Mohammad, and P. Tavner, "Electrical \& mechanical diagnostic indicators of wind turbine induction generator rotor faults," Renewable Energy, vol. 131, pp. 1424, 22019.

[9] G. Singh and V. Naikan, "Detection of half broken rotor bar fault in VFD driven induction motor drive using motor square current MUSIC analysis," Mechanical Systems and Signal Processing, vol. 110, pp. 333348, 92018.

[10] J. Burriel-Valencia, R. Puche-Panadero, J. Martinez-Roman, A. SapenaBano, and M. Pineda-Sanchez, "Fault Diagnosis of Induction Machines in a Transient Regime Using Current Sensors with an Optimized Slepian Window," Sensors, vol. 18, no. 2, p. 146, 12018.

[11] M. Pineda-Sanchez, R. Puche-Panadero, J. Martinez-Roman, A. SapenaBano, M. Riera-Guasp, and J. Perez-Cruz, "Partial Inductance Model of Induction Machines for Fault Diagnosis," Sensors, vol. 18, no. 7, p. 2340, 72018 . 
a)

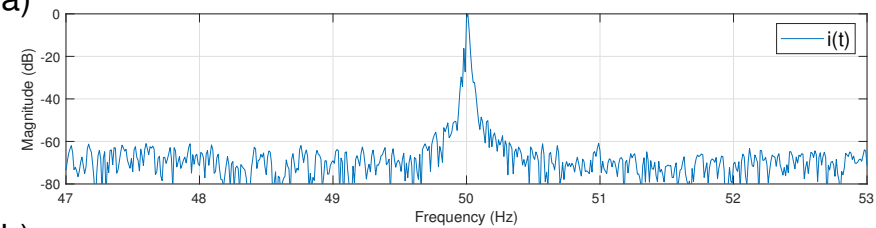

b)

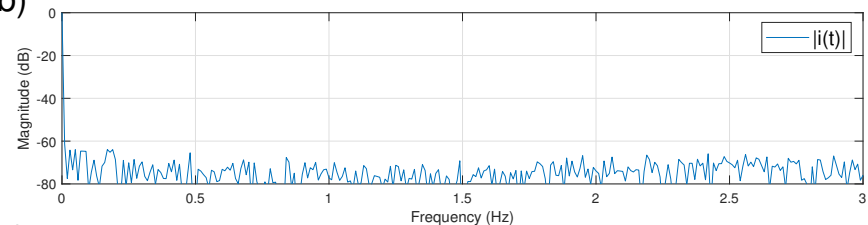

c)

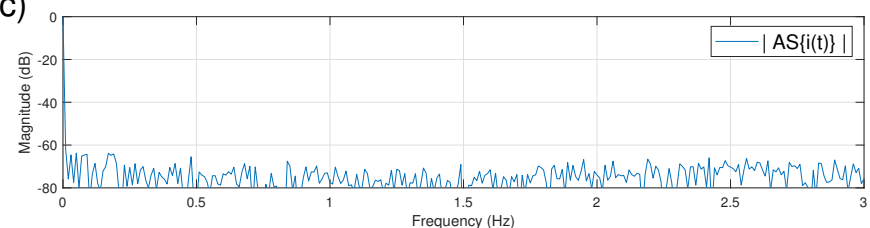

d)

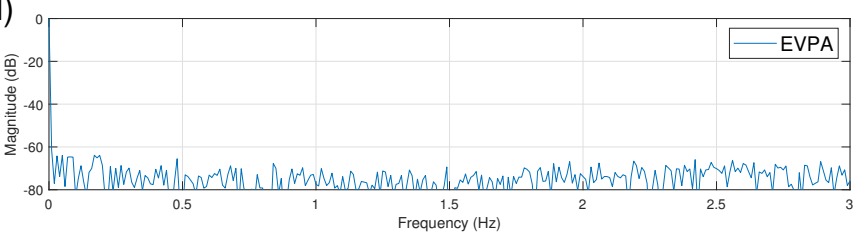

Fig. 9. Spectra of the healthy motor (no broken bars) given in Appendix A, for a motor slip of $s=0.0018$. These spectra have been obtained with the measured current (a), the proposed, rectified current (b), the modulus of the analytic signal of the current (c), and the EPVA (d). No fault harmonics appear in any spectra, so assessing a healthy motor condition.

[12] S. Choi, M. S. Haque, A. Arafat, and H. A. Toliyat, "Detection and Estimation of Extremely Small Fault Signature by Utilizing Multiple Current Sensor Signals in Electric Machines," IEEE Trans. Ind. Appl., vol. 53, no. 3, pp. 2805-2816, 52017.

[13] G. Danilo, W. G. Aguilar, D. Arcos-Aviles, and D. Sotomayor, "Broken Bar Diagnosis for Squirrel Cage Induction Motors Using Frequency Analysis Based on MCSA and Continuous Wavelet Transform," Mathematical and Computational Applications, vol. 22, no. 2, 2017.

[14] E. Artigao, S. Koukoura, A. Honrubia-Escribano, J. Carroll, A. McDonald, E. Gómez-Lázaro, E. Artigao, S. Koukoura, A. Honrubia-Escribano, J. Carroll, A. McDonald, and E. Gómez-Lázaro, "Current Signature and Vibration Analyses to Diagnose an In-Service Wind Turbine Drive Train," Energies, vol. 11, no. 4, p. 960, 42018.

[15] T. Yang, H. Pen, Z. Wang, and C. S. Chang, "Feature Knowledge Based Fault Detection of Induction Motors Through the Analysis of Stator Current Data," IEEE Trans. Instrum. Meas., vol. 65, no. 3, pp. 549558, 32016.

[16] M. Abd-el Malek, A. K. Abdelsalam, and O. E. Hassan, "Induction motor broken rotor bar fault location detection through envelope analysis of start-up current using Hilbert transform," Mechanical Systems and Signal Processing, vol. 93, pp. 332-350, 92017.

[17] M. B. Abd-el Malek, A. K. Abdelsalam, and O. E. Hassan, "Novel approach using Hilbert Transform for multiple broken rotor bars fault location detection for three phase induction motor," ISA Transactions, vol. 80, pp. 439-457, 92018.

[18] F. Cheng, L. Qu, W. Qiao, C. Wei, and L. Hao, "Fault Diagnosis of Wind Turbine Gearboxes Based on DFIG Stator Current Envelope Analysis," IEEE Trans. Sustain. Energy, pp. 1-1, 2018

[19] K. Tian, T. Zhang, Y. Ai, W. Zhang, K. Tian, T. Zhang, Y. Ai, and W. Zhang, "Induction Motors Dynamic Eccentricity Fault Diagnosis Based on the Combined Use of WPD and EMD-Simulation Study," Applied Sciences, vol. 8, no. 10, p. 1709, 92018.

[20] L. Maraaba, Z. Al-Hamouz, M. Abido, L. Maraaba, Z. Al-Hamouz, and M. Abido, "An Efficient Stator Inter-Turn Fault Diagnosis Tool for Induction Motors," Energies, vol. 11, no. 3, p. 653, 32018.

[21] F. Piltan and J.-M. Kim, "Bearing Fault Diagnosis by a Robust HigherOrder Super-Twisting Sliding Mode Observer," Sensors, vol. 18, no. 4, p. $1128,42018$.

[22] T. Berredjem and M. Benidir, "Bearing faults diagnosis using fuzzy expert system relying on an Improved Range Overlaps and Similarity method," Expert Systems with Applications, vol. 108, pp. 134-142, 10 2018.

[23] B. Bengherbia, M. Ould Zmirli, A. Toubal, and A. Guessoum, "FPGAbased wireless sensor nodes for vibration monitoring system and fault diagnosis," Measurement, vol. 101, pp. 81-92, 42017.

[24] R. A. Lizarraga-Morales, C. Rodriguez-Donate, E. Cabal-Yepez, M. Lopez-Ramirez, L. M. Ledesma-Carrillo, and E. R. FerruchoAlvarez, "Novel FPGA-based Methodology for Early Broken Rotor Bar Detection and Classification Through Homogeneity Estimation," IEEE Trans. Instrum. Meas., vol. 66, no. 7, pp. 1760-1769, 72017.

[25] P. A. Panagiotou, I. Arvanitakis, N. Lophitis, J. A. Antonino-Daviu, and K. N. Gyftakis, "A new approach for broken rotor bar detection in induction motors using frequency extraction in stray flux signals," IEEE Transactions on Industry Applications, vol. 55, no. 4, pp. 3501-3511, July 2019.

[26] M. Skowron, M. Wolkiewicz, T. Orlowska-Kowalska, and C. T. Kowalski, "Effectiveness of selected neural network structures based on axial flux analysis in stator and rotor winding incipient fault detection of inverter-fed induction motors," Energies, vol. 12, no. 12, 2019. [Online]. Available: https://www.mdpi.com/1996-1073/12/12/2392

[27] A. Glowacz and Z. Glowacz, "Diagnosis of the three-phase induction motor using thermal imaging," Infrared Physics \& Technology, vol. 81, pp. 7-16, 32017.

[28] Y. Xie, J. Guo, P. Chen, Z. Li, Y. Xie, J. Guo, P. Chen, and Z. Li, "Coupled Fluid-Thermal Analysis for Induction Motors with Broken Bars Operating under the Rated Load," Energies, vol. 11, no. 8, p. 2024, 82018.

[29] A. K. Samanta, A. Naha, A. Routray, and A. K. Deb, "Fast and accurate spectral estimation for online detection of partial broken bar in induction motors," Mechanical Systems and Signal Processing, vol. 98, pp. 63-77, 12018.

[30] F. Cheng, J. Wang, L. Qu, and W. Qiao, "Rotor-Current-Based Fault Diagnosis for DFIG Wind Turbine Drivetrain Gearboxes Using Frequency Analysis and a Deep Classifier," IEEE Trans. Ind. Appl., vol. 54, no. 2, pp. 1062-1071, 32018

[31] D. G. Dorrell and K. Makhoba, "Detection of Inter-Turn Stator Faults in Induction Motors Using Short-Term Averaging of Forward and Backward Rotating Stator Current Phasors for Fast Prognostics," IEEE Trans. Magn., vol. 53, no. 11, pp. 1-7, 112017.

[32] A. Sapena-Bano, M. Pineda-Sanchez, R. Puche-Panadero, J. MartinezRoman, and Z. Kanovic, "Low-Cost Diagnosis of Rotor Asymmetries in Induction Machines Working at a Very Low Slip Using the Reduced Envelope of the Stator Current," IEEE Trans. Energy Convers., vol. 30, no. 4, pp. 1409-1419, 122015

[33] P. A. Delgado-Arredondo, D. Morinigo-Sotelo, R. A. Osornio-Rios, J. G. Avina-Cervantes, H. Rostro-Gonzalez, and R. de Jesus RomeroTroncoso, "Methodology for fault detection in induction motors via sound and vibration signals," Mechanical Systems and Signal Processing, vol. 83, pp. $568-589,2017$.

[34] F. Dalvand, S. Dalvand, F. Sharafi, and M. Pecht, "Current Noise Cancellation for Bearing Fault Diagnosis Using Time Shifting," IEEE Trans. Ind. Electron., vol. 64, no. 10, pp. 8138-8147, 102017.

[35] Z. Feng, X. Chen, and M. J. Zuo, "Induction Motor Stator Current AM-FM Model and Demodulation Analysis for Planetary Gearbox Fault Diagnosis," IEEE Trans. Ind. Informat., vol. 15, pp. 2386-2394, 42018.

[36] J. de Jesus Rangel-Magdaleno, H. Peregrina-Barreto, J. M. RamirezCortes, P. Gomez-Gil, and R. Morales-Caporal, "FPGA-Based Broken Bars Detection on Induction Motors Under Different Load Using Motor Current Signature Analysis and Mathematical Morphology," IEEE Trans. Instrum. Meas., vol. 63, no. 5, pp. 1032-1040, 52014.

[37] J.-H. Jung, J.-J. Lee, and B.-H. Kwon, "Online Diagnosis of Induction Motors Using MCSA,' IEEE Trans. Ind. Electron., vol. 53, no. 6, pp. 1842-1852, 122006

[38] S. Lu, Q. He, T. Yuan, and F. Kong, "Online Fault Diagnosis of Motor Bearing via Stochastic-Resonance-Based Adaptive Filter in an Embedded System,” IEEE Syst., Man, Cybern. Mag., vol. 47, no. 7, pp. 1111-1122, 72017.

[39] Z. Liu, X. Yin, Z. Zhang, D. Chen, and W. Chen, "Online Rotor Mixed Fault Diagnosis Way Based on Spectrum Analysis of Instantaneous Power in Squirrel Cage Induction Motors," IEEE Trans. Energy Convers., vol. 19, no. 3, pp. 485-490, 92004.

[40] R. Puche-Panadero, M. Pineda-Sanchez, M. Riera-Guasp, J. RogerFolch, E. Hurtado-Perez, and J. Perez-Cruz, "Improved Resolution of the MCSA Method Via Hilbert Transform, Enabling the Diagnosis of 
Rotor Asymmetries at Very Low Slip," IEEE Trans. Energy Convers., vol. 24, no. 1, pp. 52-59, 32009.

[41] B. Xu, L. Sun, L. Xu, and G. Xu, "Improvement of the Hilbert Method via ESPRIT for Detecting Rotor Fault in Induction Motors at Low Slip," IEEE Trans. Energy Convers., vol. 28, no. 1, pp. 225-233, 32013.

[42] B. Bessam, A. Menacer, M. Boumehraz, and H. Cherif, "Detection of broken rotor bar faults in induction motor at low load using neural network," ISA Transactions, vol. 64, pp. 241-246, 92016.

[43] V. Climente-Alarcon, A. Arkkio, and J. Antonino-Daviu, "Study of thermal stresses developed during a fatigue test on an electrical motor rotor cage," International Journal of Fatigue, vol. 120, pp. 56 - 64, 2019. [Online]. Available: http://www.sciencedirect.com/science/article/pii/S0142112318306996

[44] T. A. Garcia-Calva, D. Morinigo-Sotelo, A. Garcia-Perez, D. CamarenaMartinez, and R. de Jesus Romero-Troncoso, "Demodulation technique for broken rotor bar detection in inverter-fed induction motor under non-stationary conditions," IEEE Transactions on Energy Conversion, vol. 34, no. 3, pp. 1496-1503, Sep. 2019.

[45] M. Pineda-Sanchez, R. Puche-Panadero, M. Riera-Guasp, J. PerezCruz, J. Roger-Folch, J. Pons-Llinares, V. Climente-Alarcon, and J. A Antonino-Daviu, "Application of the Teager-Kaiser Energy Operator to the Fault Diagnosis of Induction Motors," IEEE Trans. Energy Convers., vol. 28, no. 4, pp. 1036-1044, 122013.

[46] V. F. Pires, M. Kadivonga, J. Martins, and A. Pires, "Motor square current signature analysis for induction motor rotor diagnosis," Measurement, vol. 46, no. 2, pp. 942-948, 22013.

[47] S. M. A. Cruz, A. J. Marques Cardoso, "Rotor Cage Fault Diagnosis in Three-Phase Induction Motors by Extended Park's Vector Approach," Electric Machines \& Power Systems, vol. 28, no. 4, pp. 289-299, 4 2000.

[48] O. Guellout, A. Rezig, S. Touati, and A. Djerdir, "Elimination of broken rotor bars false indications in induction machines," Mathematics and Computers in Simulation, p. In press, 2019. [Online]. Available: http://www.sciencedirect.com/science/article/pii/S0378475419302083

[49] I. Culbert and J. Letal, "Signature Analysis for Online Motor Diagnostics: Early Detection of Rotating Machine Problems Prior to Failure," IEEE Ind. Appl. Mag., vol. 23, no. 4, pp. 76-81, 72017. 